\title{
Phenol Sensing Studies by 4-Aminoantipyrine Method-A Review
}

\author{
Chitra Varadaraju, G Tamilselvan, IVMV Enoch and Paulraj Mosae Selvakumar* \\ Department of Chemistry, School of Science and Humanities, Karunya University, India
}

Submission: December 22, 2017; Published: January 26, 2018

"Corresponding author: Paulraj Mosae Selvakumar, Department of Chemistry, School of Science and Humanities, Karunya University, Coimbatore 641114, Tamil Nadu, India, Email: pmosae@gmail.com

\begin{abstract}
4-aminoantipyrine is the most widely used analytical reagent for the estimation of phenol. Phenolic compounds were determined by buffering the sample to a $\mathrm{pH}$ of 10.0 and adding 4-aminoantipyrine to produce a yellow or amber colored complex in the presence of ferricyanide ion. The colour is intensified through extraction of the complex into chloroform. Measurement of this colour quantitatively determines the phenol concentration of the sample. In this review, we discuss the usage of 4-aminoantipyrine as analytical reagent for the identification and estimation of phenolic compounds present in any of the samples reported. This review focuses on the use of 4-aminoantipyrine in phenol sensing studies from early 1980 s to till date.
\end{abstract}

Keywords : 4-Aminoantipyrine; Phenol sensing; Peroxide; Cholesterol; Uric acid; Analytical reagent

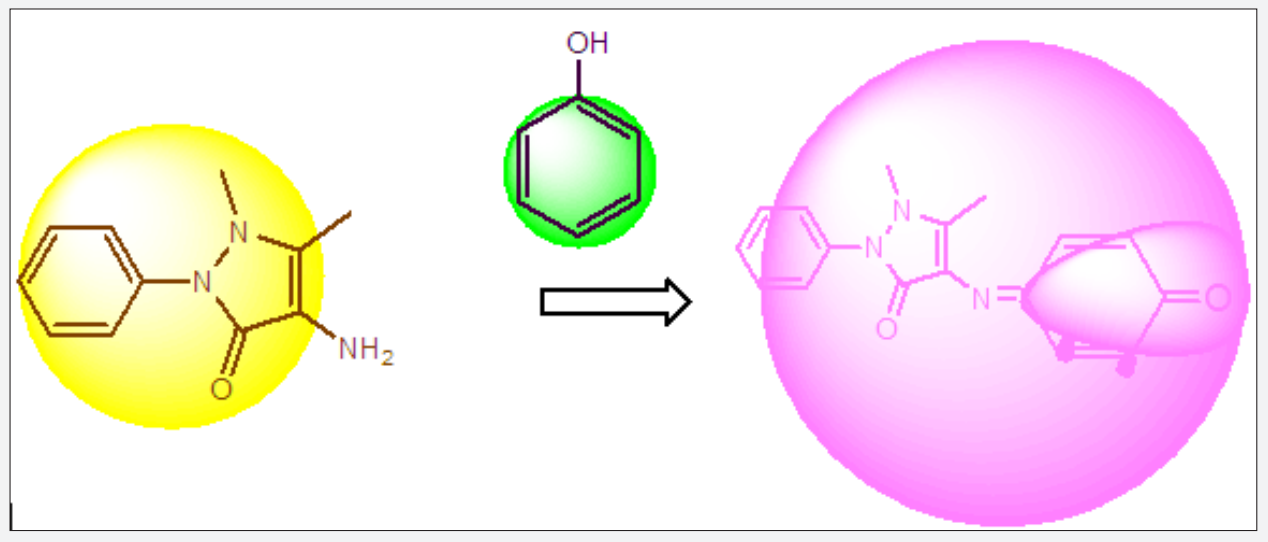

\section{Introduction}

Phenolic compounds are secondary products which posses and aromatic ring bearing a hydroxyl substituent and most are of plant origin. In keeping with their chemical diversity, phenolics play a variety of important roles in the plant. Many phenolic compounds are attracting the attention of food and medical scientists because of their antioxidative, antiinflammatory, antimutagenic, and anticarcinogenic properties and their capacity to modulate some key cellular enzyme functions. Phenols are one of the major groups of nonessential dietary components appearing in vegetable foods. The bioactivity of phenolics may be related to their antioxidant behaviour, which is attributed to their ability to chelate metals, inhibit lipoxygenase and scavenge free radicals [1]. Phenol is a normal metabolic product, excreted in quantities up to $40 \mathrm{mg} / \mathrm{L}$ in human urine.
Phenolic compounds are of environmental interest and are currently formed in different processes: industrial, biochemical, pesticide formation, etc [2].

Quantitative and qualitative detection of biologically important cations and anions is of great interest to scientists nowa-days. Many analytical methods are available to detect metal ions, but require tedious procedures, pre-treatment processes and costly equipments. To overcome this, chemosensors are designed and developed for detection of cations, anions, amino acids, etc. Development of chemosensors received much attention recently due to its fast response, simple procedures and low cost apparatus [3-5]. 4-Aminoantipyrine, a metabolite of antipyrine is an anti-inflammatory, anti-pyretic agent. It readily forms metal complexes due to its amino nitrogen, a strong 
coordination site. 4-aminoantipyrine forms Schiff bases when treated with aldehydes/ketones, which are used in chemosensing applications [6]. 4-aminoantipyrine derivatives were developed as effective chemosensors for detection of cations and anions $[7,8]$.

The use of 4-aminoantipyrine for estimation of phenol was first proposed by Emerson in 1943. As outlined, the phenolic material was mixed with 4-aminoantipyrine in the presence of alkaline oxidising agent, preferable potassium ferricyanide, at high $\mathrm{pH}$, formed a red quinone dye. Emerson's reaction has many advantages: speedy results, easy manipulation, use of stable reagents, applicability over a wide range of concentration of phenolic materials [9]. It is also used for glucose determination in the presence of phenol and peroxidase and uric acid determination through hydrogen peroxide. Cholesterol esters were hydrolyzed to free cholesterol by cholesterol ester hydrolase. The free cholesterol produced was oxidized by cholesterol oxidase to cholest-4-en-3-one with the simultaneous production of hydrogen peroxide and oxidatively coupled with 4-aminoantipyrine and phenol in the presence of peroxidise to yield a chromogen with maximum absorption at $500 \mathrm{~nm}$.

\section{4-Aminoantipyrine as Analytical reagent}

Analytical reagent for Phenol: (Scheme 1) The use of 4-aminoantipyrine for the colorimetric determination of phenolic materials was first proposed by Emerson in 1943. Hence 4-aminoantipyrine was also known as Emerson's reagent. He proposed that the reaction consisted of mixing the phenolic material, 4-aminoantipyrine and an alkaline oxidant in a solution of high $\mathrm{pH}$ to prevent the formation of quinonoid substitution product, antipyrine red proportional to phenol. Potassium ferricyanide was the oxidant used. Emerson described the structure of phenolic materials which should respond to the test. He concluded that the substitution occurred in the position para to the phenolic $\mathrm{OH}$ group. that at least one phenolic group must be present; the position para to the phenolic $\mathrm{OH}$ group must be either unsubstituted or substituted by halogen, carboxyl, sulfonic acid, hydroxyl, or methoxyl which were expelled in the reaction. Substitution in the para position by alkyl, aryl, nitro, benzoyl, nitroso or aldehyde groups blocked the reaction. He also stated that certain p-substituted halo-, hydroxyl- and methoxy- phenols during their reaction with 4-aminoantipyrine expelled the p-substituent or produce meta or ortho transfer products $[9,10]$.

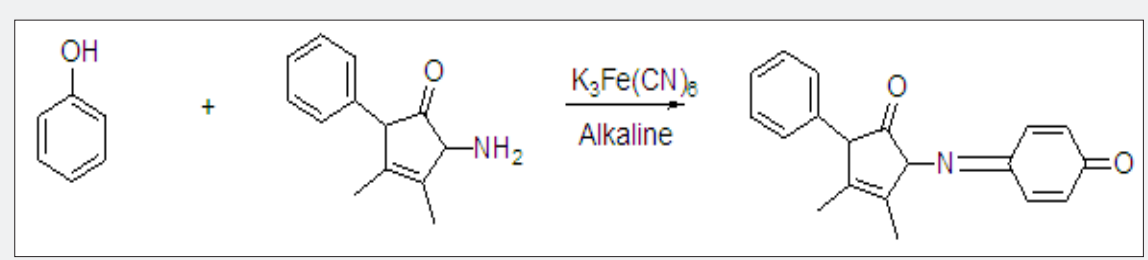

Scheme 1. Reaction between phenol and 4-aminoantipyrine.

Sensitivity of 4-aminoantipyrine method for the determination of phenolic compounds was described by Ettinger et al. [11]. Evaluation of reaction variables and a procedure sensitive to detect $1 \mathrm{ppb}$ concentration of phenol was described. Sensitivity of the reaction depends on the concentration of the dye formed. It was found that chlorinated hydrocarbons like chloroform, ethylene dichloride, carbon tetrachloride and tetra chloroethane was used to extract the dye from the reaction mixture. Chloroform appeared to be the best of chlorinated hydrocarbons in dye extraction as reported early by Emerson due to its extraction efficiency, intensity of the color of the extract, stability of the extracted color, cost, availability and toxicity [9]. The red aqueous dye was extracted with chloroform to yield orange or yellow solution. The maximum stability of the color produced appeared to be in the $\mathrm{pH}$ range of 9.4-10.2 [11]. Determination of catechols in the presence of other phenols could be possible when atmospheric oxygen was used as oxidant instead of potassium ferricyanide [12].

The interferences of the oxidants $\mathrm{ClO}-, \mathrm{ClO}_{2}^{-}, \mathrm{ClO}_{3}^{-}, \mathrm{MnO}_{4}^{-}$, $\mathrm{H}_{2} \mathrm{O}_{2}, \mathrm{Cr}_{2} \mathrm{O}_{7}{ }^{2}-\mathrm{S}_{2} \mathrm{O}_{8}{ }^{2}$ - and $\mathrm{NO}_{3}$ - with 4-aminoantipyrine in the determination of phenols was minimized by the addition of sodium arsenite, followed by distillation. Phenol was oxidised by these interferences and produced low results. The results by the 4-AAP method without distillation showed that ClOand $\mathrm{MnO}_{4}$ - interfere markedly, $\mathrm{H}_{2} \mathrm{O}_{2}$ interferes less strongly, $\mathrm{ClO}_{2}$ - and $\mathrm{S}^{2} \mathrm{O}_{8}{ }^{2}$ - do not interfere unless present in large amount while $\mathrm{ClO}_{3}^{-}, \mathrm{Cr}_{2} \mathrm{O}_{7} 2$ - and $\mathrm{NO}_{3}$ - hardly interfere at all. When the solutions were distilled, the interference of $\mathrm{ClO}_{2}^{-}, \mathrm{H}_{2} \mathrm{O} 2$ and $\mathrm{S}_{2} \mathrm{O}_{8}{ }^{2}$ - increased markedly [13]. The interference of sulphite with 4-aminoantipyrine method for phenol without distillation and with distillation in the absence and presence of copper (II) sulphate was studied. The study revealed that sulphite repressed the color development in all these procedures. Without chloroform extraction, the maximum amount of sulphite in these procedures was 15,10 and $20 \mathrm{mg} / 100 \mathrm{ml}$. For the extraction method, the limits were 4,4 and $10 \mathrm{mg} / 100 \mathrm{ml}$. Copper (II) sulphates catalyzed the air-oxidation of sulphite. It was demonstrated that phenol could be determined in the presence of large amounts of sulphite by treating with sulphide to form polythionates and thiosulphate; excess of sulphite was removed with copper (II) sulphate, sulphuric acid was added, then phenol was distilled and then 4-AAP method was applied [14].

Replacement of potassium hexacyanoferrate (III) as the oxidizing agent in the Emerson's reaction by using chloride as the oxidizing agent was studied. Replacement was done because of the large number of by-products that absorb in the 
UV region. The HPLC separation of dyes did not reveal many number of reaction by-products when silver chloride was used as the oxidizing agent. Optimum conditions for the oxidative coupling between 4-AAP and the phenol in the presence of silver chloride were studied. The reaction time was fixed at $40 \mathrm{~min}$ and the temperature was at $40^{\circ} \mathrm{C}$. Also the coupling of phenols with 4-AAP in the presence of $\mathrm{AgCl}$ was not instantaneous, but required 30-60 min for completion. Presence of dextrin colloid protector and the introduction of chloroform extractant at the beginning of the reaction improved the efficiency of the $\mathrm{AgCl}$ oxidant. Emerson's reaction yields by para-substituted phenols were lower than those by the corresponding non-para substituted phenols when potassium hexacyanoferrate (III). But when silver chloride was used as the oxidizing agent, decrease in yield was enhanced. Hence using $\mathrm{AgCl}$, UV detection of the derivative was possible and the selectivity in the determination of para-substituted phenols was improved [15].

The determination of monohydric phenols by highperformance liquid chromatography (HPLC) after derivatization with 4-aminoantipyrine had been studied [16]. The HPLC determination of 1-naphthol and 2-naphthol after derivatization with 4-aminonatipyrine showed two main coloured products for each naphthols. Determination of 1-naphthol was performed in the $0.05-10$ ppm concentration range, but instability of 2-naphthol products made their quantitative determination difficult. A spectrophotometric method for carbaryl determination based on the 4-AAP derivatization of 1-naphthol had developed in 1982. 1-naphthol was the major degradation product of the insecticide carbaryl. The applicability of the method was checked by determining the carbaryl content in commercial powder formulations and residues in apples owing to its high selectivity and sensitivity [17]. Colorimetric determination of propoxur in pesticide formulations and water samples was based on the alkaline hydrolysis of pesticide and the resultant phenol was reacted with 4-aminoantipyrine in the presence of an oxidizing agent. The resultant orange color dye was extracted into chloroform and the absorbance was measured at $472 \mathrm{~nm}$. Extraction of the dye from aqueous solution into small quantity of organic solvent increased the sensitivity of the method by several-fold compared to other reported methods and also the stability of the coloured dye in organic solvents was more when compared with the aqueous phase [18].

Determination of urinary phenolic compounds appeared to be a useful tool for patients with hyperthyroidism, diabetes mellitus and catecholamine-producing tumor. Urine sample, 4-aminoantipyrine in carbonate-bicarbonate buffer and potassium ferricyanide solution were mixed together and the obtained quinoneimine dye was measured [19]. Estimation of phenyl phosphatase was done by measuring the liberated phenol and allowed it to react with 4-aminoantipyrine in the presence of alkaline oxidising agent. This method did not require removal of proteins as other methods, since 4-AAP did not react with plasma proteins and their removal was unnecessary when phosphatase was estimated [20]. 4-Aminoantipyrine was used to estimate the amount of pentachlorophenol (PCP) present in wood. PCP, a registered fungicide used as a preservative in wood products and in wood-based packaging materials. When present at elevated levels it could taint foods and beverages and impart pungent offflavours in food materials. Therefore rapid screening methods were required to identify wood-based materials containing high percentage of PCP. The acidic hydroxyl group of PCP enabled its coupling with 4-AAP under acidic $\mathrm{pH}$. This reaction is relatively specific since lower substituted chlorophenols and nonchlorinated phenols react with 4-AAP under alkaline condition. Kinetic studies showed that maximum dye formation was occurred within $2 \mathrm{~min}$ of persulfate oxidation and then gradually decreased over time. The PCP-antipyrine dye complex was stable up to 90 min followed by extraction in toluene [21].

A cost-effective strategy based on ELISA kit for 4-nitrophenol was used for the characterization of waste water sample of primary and secondary sewage effluent using 4-aminoantipyrine method [22]. Six newly synthesized 4-aminopyrazolones when reacted with phenolic compounds form coloured quinonoid products. In all cases, these products were analogous to the one formed by reaction between 4-AAP and the phenolic compounds. Diaminopyrazolones did not form diquinone-imide structures. The amine in the para position of the pyrazolone ring reacted more readily with phenolic compound without allowing the formation of other quinonoid by-products. The 4-amino group is more reactive due to its participation in a conjugates system present in the pyrazolone ring and stabilized the intermediate cationic forms or radicals. The numerous by-products of these reactions were colorless and therefore do not interfere in the method. The p-substituted phenols formed substitution products during their reaction with 4-aminopyrazolones [23]. Condensation of 4-APP with phenolic substances in the presence of an alkaline oxidizing agent to yield a coloured product was proposed, but the method was completely manual and consumed large amount of reagents. Hence flow analysis known to be fast, precise and inexpensive enhances selectivity and sensitivity to allow multiple analysis and easy to automate compared to manual or batch methods. Two important flow systems were FIA and SIA. The main advantages of SIA over FIA were: low reagent consumption, minimum waste generation, ability to perform several difference measurements coupled with various modes of detection without the need to reconfigure the flow manifold. 4-AAP method when carried out in batch, the reaction requires extraction of the coloured substance formed into chloroform prior to spectroscopic measurement. The use of FIA should overcome the need of separation by virtue of the accurate timing.

The use of transient signal measured from a baseline, characteristic of FIA may contribute to minimise the influence of a coloured reagent such as hexacyanoferrate (III). FIA method did not require solvent extraction step. A new flow-injection analytical procedure was proposed for the determination of total amount of polyphenols in wines; the method was based on the 
formation of a coloured complex between 4-aminoantipyrine and phenols in the presence of oxidizing agent. The oxidizing agents used were hexacyanoferrate (III) (HCF), peroxodisulfate (PDS) and tetroxoiodate (VII) (TOI). The establishment of this alternate procedure with a different chemical background enabled simple and more selective determination of total polyphenols. Among the different oxidizing reagents used, sensitivity and repeatability were best when HCF was used. The sensitivity was 2.1 and 3.1 times higher than those of PDS and TOI. Interference from several compounds present in wines was also evaluated. For reducing and non-reducing sugars, no interference was detected. The accuracy of results obtained by use of this FIA method was good because errors relative to those obtained in other methods were below $5 \%$ for most samples [24].

A flow-injection method was proposed for the determination of salbutamol. Salbutamol, employed as bronchodilator for the treatment of asthma and chronic obstructive pulmonary disease. It was also used to arrest premature labor in pregnancy [25]. Direct UV measurement of salbutamol was difficult since combined spectra of preservatives, sweetners and colorants gave additional interferences. This method could be used in routine pharmaceutical quality control without the need of resorting to more expensive and complex procedures. The method involved the condensation of salbutamol with 4-aminoantipyrine in the presence of hexacyanoferrate (III) in alkaline medium, producing a coloured quinoneimide and was detected absorptiometrically at $500 \mathrm{~nm}$ [26]. Ritodrine hydrochloride was used as uterine relaxant, inhibiting the contractility of uterine smooth muscle resulted in the arrest of premature labor. Several methods have been reported for the determination of ritodrine hydrochloride which includes HPLC, fluorimetric and spectrophotmetric methods. All these methods were expensive, low sensitivity, low sample throughput, long incubation time, etc. But condensation of 4-APP with phenolic substances in the presence of an alkaline oxidizing agent to yield a coloured product was proposed, but the method was completely manual and consumed large amount of reagents. Hence a flow analysis system, particularly sequential injection analysis method was proposed [27].

Analytical reagent for hydrogen peroxide: (Scheme 2) Glucose oxidase/peroxidase system was widely used for determination of glucose in blood. In this reaction, glucose was converted to gluconic acid and $\mathrm{H}_{2} \mathrm{O}_{2}$, catalyzed by glucose oxidase. The formed $\mathrm{H}_{2} \mathrm{O}_{2}$ reacted with 4-aminoantipyrine and phenol, produced a red color dye in the presence of horseradish peroxidase (HRP) [28]. Kinetic determination of glucose in fruit drinks and carbonated beverages using glucose oxidase and horseradish peroxidase were described [29]. 4-aminoantipyrine colorimetry method was developed for the detection of minute quantities of residual hydrogen peroxide in noodles, fish paste, dried fish and herring roe. Hydrogen peroxide was stabilized with potassium bromated and extracted with cold methanol. The methanol extract was diluted with phosphate buffer, protein was eliminated with zinc sulphate and the extract was reacted with phenol, 4-AAP and peroxidase to the stable quinoneimine dye [30]. Glucose oxidase (GOX) was specific for $\beta$-D-Glucose, the coupling reaction was completely non-specific and many oxidizing agents would give a colored product with phenol4-aminoantipyrine system. This reaction could be used for the determination of hydroperoxide in seafoods and liquid hydroperoxides in oils and fats (soybean oil, linseed oil, olive oil, salad oil, butter and lard) [31].

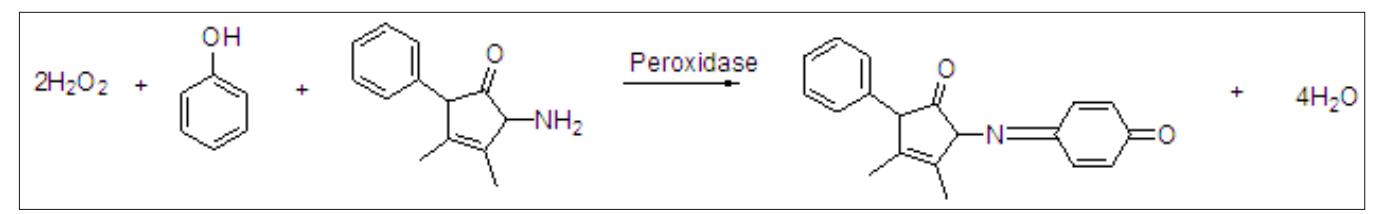

Scheme 2. Reaction between 4-aminoantipyrine and $\mathrm{H}_{2} \mathrm{O}_{2}$.

Determination of hydrogen peroxide scavenging activity of plant extracts was evaluated by measuring the disappearance of $\mathrm{H}_{2} \mathrm{O}_{2}$ at wavelength of $230 \mathrm{~nm}$. A simple and rapid colorimetric assay was developed where plant extracts were introduced to $\mathrm{H}_{2} \mathrm{O}_{2}$, phenol and 4-aminoantipyrine reaction system in the presence of horseradish peroxidase (HRP). This reaction yields a quinoneimine chromogen which can be measured at $504 \mathrm{~nm}$. Decrease in the colour intensity reflects the $\mathrm{H}_{2} \mathrm{O}_{2}$ scavenged by the plant material [32]. A simple colorimetric method for determination of hydrogen peroxide in plant material was based on producing a stable red product in reaction with 4-aminoantipyrine and phenol in the presence of peroxidises [33]. An enzymatic method was described for determination of total serum cholesterol by use of a single aqueous reagent. Cholesterol esters were hydrolyzed to free cholesterol by cholesterol ester hydrolase. The free cholesterol produced was oxidized by cholesterol oxidase to cholest-4-en-3-one with the simultaneous production of hydrogen peroxide and oxidatively coupled with 4-aminoantipyrine and phenol in the presence of peroxidise to yield a chromogen with maximum absorption at $500 \mathrm{~nm}[34]$.

Instead of phenol/4-aminoantipyrine reagent, 4-hydroxybenzoate/4-aminoantipyrine chromogenic system was used in the enzymatic determination of serum cholesterol. This system has the advantage of increasing the stability of the singe working reagent, which could be stored for several months in solution [35]. A manual method was described for determining the hydrogen peroxide produced from uric acid with uricase. 2,4,6-tribromophenol was coupled with 4 -aminoantipyrine by peroxidise oxidative coupling. This meth- 
od was reproducible and many substances encountered in serum did not interfere in uric acid determination. This method required deproteinization of the sample [36]. A new enzymatic method for serum uric acid determination was proposed by measuring hydrogen peroxide produced from uric acid by uricase when coupled with p-hydroxybenzoate and 4-aminoantipyrine. Disadvantages in other methods like sample deproteinization, long reaction times, use of carcinogenic dyes were overcome by this method. Uric acid on oxidative deacarboxylation by uricase forms allantoin and peroxide. The formed peroxide coupled with p-hydroxybenzoate and 4-aminoantipyrine yields quinoneimine chromophore. The change in absorbance at $500 \mathrm{~nm}$ when the quinoneimide chromophore formed was directly proportional to the uric acid concentration of the sample [37].

Amberlite IRA 900 anion-exchange resin modified with manganese-tetrakis(sulphophenyl)-porphine had been used as a catalyst instead of peroxidase for the determination of hydrogen peroxide. But large amount of phenol was used since phenol was adsorbed on the modified resin. Hence N,N-diethylaniline was used in the reaction. The results obtained indicated that DEA-AAP system was better than the phenol-AAP system for the determination of hydrogen peroxide with the modified resin as catalyst [38]. dye

$\mathrm{H}_{2} \mathrm{O}_{2}+\mathrm{N}, \mathrm{N}$-diethylaniline +4 -aminoantipyrine quinonoid

Immobilization of enzymes likes glucose oxidase (GOx) on inorganic carried materials were widely used technique for producing chemical sensors. GOx was immobilized on chalcogenide and silver halide fibre surfaces. Chemical modification on fibre surfaces act as an enzyme carried and the so-treated fibre materials were tested for enzymatic assays [39]. Examination of monoamine oxidase and copper amine oxidase enzymatic activities in the presence of suitable amine substrate were carried out. Amine oxidase enzymes generated hydrogen peroxide undergoes peroxidase-dependent oxidation of 4-aminoantipyrine. Interaction with vanillic acid produced a red quinoneimide dye, monitored at $498 \mathrm{~nm}$ [40].

Analytical reagent for other molecules: A kinetic-catalytic method for the determination of iron based on its catalytic effect on the oxidative coupling of 4-aminoantipyrine (AA) with $\mathrm{N}, \mathrm{N}$ dimethylaniline (DMA) to form an indamines dye in the presence of hydrogen peroxide was described by Nakano et al. [41]. Also they have proposed a method for the determination of vanadium (IV, V) using oxidative coupling of 4-aminoanitpyrine with $\mathrm{N}$, $\mathrm{N}$-diemthylaniline in the presence of bromated [41,42]. It was observed that the dye forming reaction was also catalysed by traces of iron (II, III) in the presence of hydrogen peroxide as an oxidant and acetate as an activator. The rate of oxidative coupling of AA with DMA by hydrogen peroxide was slow, but rapid in the presence of traces of iron (II, III) [43].

The feasibility of 4-AAP as a chromogenic agent for the colorimetric determination of aromatic amines were studied.
The effect of $\mathrm{pH}$, reducing and interfering compounds were also evaluated. The method was not sensitive to chloro-, nitro-, p-substituted anilines and polycyclic aromatic amines [44]. A colorimetric method was developed for the determination of tetracycline and oxytetracycline and their dosage forms. These method depends on the reaction with 4-aminoantipyrine to produce a red antipyrine dye in the presence of an alkaline oxidising agent and Charge transfer complex formation $[45,46]$. Further, Colorimetric determination of phenylephrine, propoxur and 3-methylphenyl $\mathrm{N}$-methylcarbamate also reported using 4-Aminoantipyrine as reagent [47-49].

\section{Conclusion}

4-Aminoantipyrine has been known to possess potential diverse applications in biological, clinical, analytical, and pharmacological areas. This area of research has culminated in a deeperunderstandingofavarietyofapplicationsincludingclinical, analytical, industrial and catalytical roles. 4-Aminoantipyrine is acting as a versatile analytical reagent for the determination phenols in real time analysis. 4-Aminoantipyrine not only used for the colorimetric determination of phenolic compounds but also used as analytical reagent for the determination of hydrogen peroxide, cholesterol, aromatic amines, uric acid , phenylephrine , propoxur and 3-methylphenyl N-methylcarbamate, Tetracycline and Oxytetracycline.

\section{References}

1. Martinez-Valverde, Periago MJ, Ros G (2000) Nutritional importance of phenolic compounds in the diet. Arch Latinoam Nutr 50(1): 5-18.

2. M La Farre, A Oubina, MP Marco, A Ginebreda, L Tirapu, et al. (1999) Evaluation of 4-Nitrophenol ELISA Kit for Assessing the Origin of Organic Pollution in Wastewater Treatment Works. Environ Sci Technol 33(21): 3898-3904.

3. GT Selvan, V Chitra, Enoch IVMV, PM Selvakumar (2018) Development of fluorescent chemosensor towards sensing and separation of $\mathrm{Mg}^{2+}$ ions in chlorophyll and hard water. New J of Chem.

4. M Sumithra, R Sivaraj, GT Selvan, PM Selvakumar, IVMV Enoch (2017) $\mathrm{Ca}^{2+}$ ion sensing by a piperidin-4-one derivative and the effect of $\beta$-cyclodextrin complexation on the sensing. J of Luminesc 185: 205211.

5. G Tamil Selvan, Chitra Varadaraju, VMVEnoch, P Mosae Selvakumar, E Suresh (2017) 1-(1-Hydroxynapththalen-2-yl) Ethanone: Crystal structure, Photo Physical study and Turn OFF molecular switch with $\mathrm{Cu}(\mathrm{II})$ Ion. New J of Chem 42: 902-909.

6. PM Selvakumar, E Suresh, PS Subramanian (2007) Synthesis, spectral characterization and structural investigation on some 4-aminoantipyrine containing Schiff base $\mathrm{Cu}(\mathrm{II})$ complexes and their molecular association. Polyhedron 26(4): 749-756.

7. GT Selvan, M Kumaresan, R Sivaraj, IVMV Enoch, PM Selvakumar (2016) Isomeric 4- aminoantipyrine derivatives as fluorescent chemosensors of $\mathrm{Al}^{3+}$ ions and their molecular logic behavior. Sens Actuators B 229: 181-189.

8. Premnath D, Selvakumar PM, Ravichandiran P, Selvan GT, Indiraleka M, et al. (2016) Synthesis and spectroscopic characterization of fluorescent 4-aminoantipyrine analogues: Molecular docking and in vitro cytotoxicity studies. Spectrochim ActaA Mol Biomol Spectrosc 153: $118-123$ 
9. E Emerson (1943) The condensation of aminoantipyrine. II. A new color test for phenolic compounds. J Org Chem 8(5): 417-428.

10. RWMartin (1949) Rapid colorimetric estimation of phenol. Anal Chem 21(11): 1419-1420.

11. MB Ettinger, CC Ruchhoft, RJ Lishka (1951) Sensitive 4-aminoantipyrine method for phenolic compounds. Anal Chem 23(12): 1783-1788.

12. Larue TA, Blakley ER (1964) Spectrophotometric determination of catechols with 4-aminoantipyrine. Anal Chim Acta 31: 400-403.

13. Norwitz G, Farino J, Keliher PN (1979) Interference of oxidants in the determination of phenol by the 4-aminoantipyrine and ultraviolet ratio spectroscopic methods. Anal Chem 51: 1632-1637.

14. Norwitz G, Bardsley AH, Keliher PN (1981) Determination of phenol in the presence of sulphite (sulphur dioxide) by the 4-aminoantipyrine spectro-photometric method. Anal Chim Acta 128: 251-256.

15. G Blo, F Dondi, CBighi (1984) High-performanceliquid chromatographic determination of phenols as 4-aminoantipyrine derivatives: silver chloride as oxidizing agent in the derivatization reaction. J Chromatogr 295: 231-235.

16. G Blo, F Dondi, A Betti, C Bighi (1983) Determination of phenols in water samples as 4-aminoantipyrine derivatives by high-performance liquid chromatography. J Chromatogr 257: 69-79.

17. MC Pietrogrande, G Blo, C Bighi (1985) High-performance liquid chromatographic determination of naphthols as 4-aminoantipyrine derivatives: Application to carbaryl. J Chromatogr 349: 63-68.

18. B Venkateshwarlu, K Seshaiah (1995) Sensitive spectrophotometric method for the determination of propoxur using 4-aminoantipyrine. Talanta 42(1): 73-76.

19. Yamaguchi Y, Hayashi C (1977) Determination of urinary total phenolic compounds with use of 4-aminoantipyrine: Suggested screening test for hyperthyroidism and for catecholamine-producing tumor. Clin Chem 23(11): 2151-2154.

20. Kind PRN, King EJ (1954) Estimation of plasma phosphatase by determination of hydrolysed phenol with amino-antipyrine. J Clin Path $7(4): 322-326$.

21. Gremaud E, Turesky RJ (1997) Rapid analytical methods to measure pentachlorophenol in wood. J Agri Food Chem 45(4): 1229-1233.

22. Farre ML, Oubina A, Marco MP, Ginebreda A, Tirapun L, et al. (1999) Evaluation of 4-nitrophenol ELISA kit for assessing the origin of organic pollution in wastewater treatment works. Environ Sci Technol 33: 3898-3904.

23. Y Fiamegos, C Stalikas, G Pilidis (2002) 4-aminoantipyrine spectrophotometric method of phenol analysis. Study of the reaction products via liquid chromatography with diode-array and mass spectrometric detection. Anal Chim Acta 467(1-2): 105-114.

24. Schoonen JW, Sales MGF (2002) Determination of polyphenols in wines by reaction with 4-aminoantipyrine and photometric flow-injection analysis. Anal Bioanl Chem 372: 822-828.

25. KParfitt (1999) Martindale, the complete drug reference (32 ${ }^{\text {nd }}$ edn.); Pharmaceutical Press, London.

26. I Dol, M Knochen (2004) Flow-injection spectrophotometric determination of salbutamol with 4-aminoantipyrine. Talanta 64(5): 1233-1236.

27. JF Van Staden, NW Beyene, R-I Stefan, HY Aboul-Enein (2005) Saquential injection spectrophotometric determination of ritodrine hydrochloride using 4-aminoantipyrine. Talanta 68(2): 401-405.

28. P Trinder (1969) Determination of glucose in blood using glucose oxidase with an alternative oxygen acceptor. Ann Clin Biochem 6: 2427.
29. Vasilarou AMG, Georgiou CA (2000) Enzymatic spectrophotometric reaction rate determination of glucose in fruit drinks and carbonated beverages. J Chem Educ 77: 1327-1329.

30. Y Ito, Y Tonogai, H Suzuki, S Ogawa, T Yokoyama, et al. (1981) Improved 4-aminopantipyrine colorimetry for detection of residual hydrogen peroxide in noodles, fish paste, dried fish and herring roe. J Assoc Off Anal Chem 64(6): 1448-1452.

31. I Akaza, N Aota (1990) Colorimetric determination of lipid hydroperoxides in oils and fats with microperoxidase. Talanta 37(9): 925-929.

32. CD Fernando, P Soysa (2015) Optimized enzymatic colorimetric assay for determination of hydrogen peroxide $\left(\mathrm{H}_{2} \mathrm{O}_{2}\right)$ scavenging activity of plant extracts. Methods 2: 283-291.

33. B Zhou, J Wang, Z Guo, H Tan, X Zhu (2006) A simple colorimetric method for determination of hydrogen peroxide in plant tissues. Plant Growth Regul 49(2-3): 113-118.

34. CC Allain, LS Poon, CSG Chan, W Richmond, PC Fu (1974) Enzymatic determination of total serum cholesterol. Clin Chem 20(4): 470-475.

35. F Meiattini, L Prencipe, F Bardelli, G Giannini, P Tarli (1978) The 4-hydroxybenzoate/4-aminophenozone chromogenic system used in the enzymatic determination of serum cholesterol. Clin Chem 24(12): 2161-2165.

36. P Kabasakalian, S Kalliney, A Westcott (1973) Determination of uric acid in serum, with use of uricase and a tribromophenol-aminoantipyrine chromogen. Clin Chem 19(5): 522-524.

37. RC Trivedi, L Rebar, E Berta, L Stong (1978) New enzymatic method for serum uric acid at $500 \mathrm{~nm}$. Clin Chem 24(11): 1908-1911.

38. Y Saito, M Mifune, S Nakashima, J Odo, Y Tanaka (1987) Determination of hydrogen peroxide with $\mathrm{N}, \mathrm{N}$-Diehylaniline and 4-aminoantipyrine by use of an anion-exchnage resin modified with manganestetrakis(sulphophenyl)porphine, as a substitute for peroxidise. Talanta 34(7): 667-669.

39. K Taga, S Weger, R Gobel, R Kellner (1993) Colorimetric activity assays of enzyme-modified MIR fibers. Sens Actuators B 11(1-3): 553-559.

40. A Holt, MM Palcic (2006) A peroxidae-coupled continuous absorbance plate-reader assay for flavin monoamine oxidases, copper-containing amine oxidases and related enzymes. Nat Protoc 1(5): 2498-2505.

41.S Nakano, C Yamada, M Sakai, T Kawashima (1986) Catalytic determination of nanogram amounts of vanadium by the oxidative coupling reaction of 4-aminoantipyrine with $\mathrm{N}, \mathrm{N}$-dimethylaniline. Anal Sci 2: 61-65.

42.S Nakano, M Tago, T Kawashima (1989) Catalytic determination of nanograms amounts of vanadium in natural water by flow injection analysis. Anal Sci 5: 69-72.

43.S Nakano, M Sakai, M Kurachi, T Kawashima (1994) Kinetic determination of iron based on its catalytic action on the oxidative coupling of 4-aminoantipyrine with $\mathrm{N}, \mathrm{N}$-dimethylaniline. Microchem J 49(2-3): 298-304.

44. MA El-Dib, MO Abdel-Rahman, OA Aly (1974) 4-aminoantipyrine as a chromogenic agent for aromatic amine determination in natural water. Water Res 9(5-6): 513-516.

45. M Ayad, M El-Sadek, S Mostaffa (1986) 4-Aminoantipyrine as an Analytical Reagent for the Colorimetric Determination of Tetracycline and Oxytetracycline. Analytical Letters 19(21-22): 2169-2181.

46. M El Sadek, M Ayad, S Mostaffa (1987) New Colorimetric Method for Determination of Tetracycline and Oxytetracycline Via Charge Transfer Complex Formation. Analytical Letters 20(11): 1885-1893.

47. Koshy KT, Mitchner H (1963) Colorimetric determination of phenylephrine using 4-aminoantipyrine. J Pharm Sci 52(8): 802-803. 
48. B Venkateswarlu, K Seshaiah (1995) Sensitive spectrophotometric method for the determination of propoxur using 4-aminoantipyrine. Talanta 42(1): 73-76
49. Atsushi Murano (1970) Colorimetric determination of 3-methylphenylN-methylcarbamate with 4-aminoantipyrine. Bunseki Kagaku 19(8): 1074-1077.

\section{Your next submission with Juniper Publishers will reach you the below assets}

- Quality Editorial service

- Swift Peer Review

- Reprints availability

- E-prints Service

- Manuscript Podcast for convenient understanding

- Global attainment for your research

- Manuscript accessibility in different formats ( Pdf, E-pub, Full Text, Audio)

- Unceasing customer service

Track the below URL for one-step submission https://juniperpublishers.com/online-submission.php 